\title{
The density of states in silicon nanostructures determined by space-charge-limited current measurements
}

\author{
Takahiro Matsumoto \\ Single Quantum Dot Project, ERATO, Japan Science and Technology Corporation, 5-9-9 Tokodai, \\ Tsukuba 300-2635, Japan \\ Hidenori Mimura \\ Research Institute of Electrical Communication, Tohoku University, Sendai 980-77, Japan \\ Nobuyoshi Koshida \\ Division of Electronic and Information Engineering, Tokyo University of Agriculture and Technology, \\ Koganei, Tokyo 184, Japan \\ Yasuaki Masumoto ${ }^{\text {a) }}$ \\ Single Quantum Dot Project, ERATO, Japan Science and Technology Corporation, 5-9-9 Tokodai, \\ Tsukuba 300-2635, Japan
}

(Received 18 May 1998; accepted for publication 11 September 1998)

\begin{abstract}
Space-charge-limited current (SCLC) flow was investigated as a function of applied potential and specimen thickness in nanocrystalline silicon films prepared by electrochemical anodization. From the analysis of the current-voltage $(J-V)$ characteristics in the SCLC regime, the density of states distribution near the Fermi level was determined. The agreement between the experimental $J-V$ characteristics and the theoretical curve strongly implies that the current flow is entirely controlled by localized states situated at the quasi-Fermi level. (c) 1998 American Institute of Physics. [S0021-8979(98)10823-X]
\end{abstract}

\section{INTRODUCTION}

Highly efficient visible photoluminescence (PL) from silicon nanostructures ${ }^{1}$ fabricated using electrochemical anodization has generated exciting prospects for applications both in flat-panel displays and in optical interconnections monolithically integrated into Si wafers. Many studies have aimed at achieving strong electroluminescence (EL), which enables these applications, from silicon nanostructures in a solid-state device. ${ }^{2-7}$ However, despite the vast research aimed at intense EL devices, no detailed investigation of the deep levels in this material has been reported to the best of our knowledge. Such information yields insights on critical properties, such as carrier transport for the design of optoelectronic devices.

A number of studies show that injection current can be a powerful tool for studying insulators or high resistivity materials. ${ }^{8}$ The most widespread contribution from using this tool is information about defect states in the forbidden gaplocalized defect states can strongly influence the injected current flowing in response to an applied voltage. Not only the absolute value of the current but also the actual form of the current-voltage $(J-V)$ characteristics is determined by the interaction of the injected carriers with such states. This current-voltage characteristic called space-charge-limited current (SCLC) offers a well-established and extremely sensitive method (defect state concentrations as low as $10^{12} \mathrm{~cm}^{-3}$ can be detected by this technique) to study energetic and spatial profiles of traps for current carriers. Such

a) Also with the Institute of Physics, University of Tsukuba, Tsukuba, Ibaraki 305-8571, Japan. measurements have been applied to a substantial number of materials ranging from semiconductors to molecular crystals. ${ }^{8}$

For determining the density and the distribution of deeplevel energy states, methods other than the SCLC method can be used: field-effect technique, ${ }^{9}$ capacitance-voltage methods ${ }^{10}$ and deep-level transient spectroscopy (DLTS). ${ }^{11}$ However, those three alternative techniques require highly doped material and specially designed device structures. It is, therefore, difficult and unreliable to use those three methods for measuring the density of states of Si nanostructures because carriers are depleted by the electrochemical dissolution process and many processes for the device fabrication inevitably damage the fragile Si nanostructure.

In this article, we present the results of SCLC flow as a function of applied potential and specimen thickness in nanocrystalline silicon films prepared using electrochemical anodization. From the analysis of the $J-V$ characteristics in the SCLC regime, the density of state distribution near the Fermi level in Si nanostructures was determined by a stepby-step method. ${ }^{12,13}$ We demonstrate that the SCLC method offers a powerful tool for evaluating the deep-level energy states in highly complex structures, such as porous $\mathrm{Si}$ (PS).

\section{EXPERIMENTAL RESULTS AND ANALYSIS}

Metal/PS device structures were fabricated in $p$-type silicon substrates with a resistivity of $3-5 \Omega \mathrm{cm}$, and then anodized in a solution of $\mathrm{HF}(49 \%): \mathrm{C}_{2} \mathrm{H}_{5} \mathrm{OH}=1: 1$ at a constant current density of $20 \mathrm{~mA} / \mathrm{cm}^{2}$. The porosity of the porous layer was about $80 \%$, which we confirmed by density measurements. This fabrication process produced a homoge- 


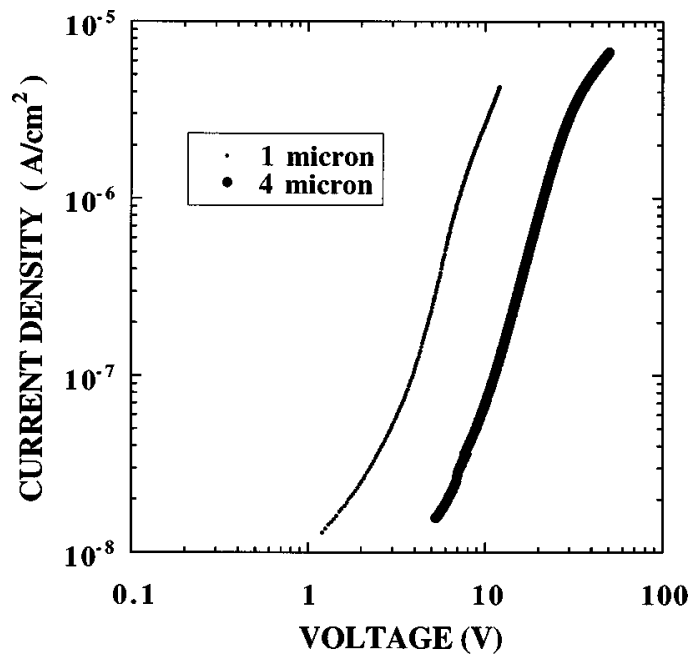

FIG. 1. Typical logarithmic current-voltage $(J-V)$ characteristics for $\mathrm{Au} / \mathrm{PS}$ junctions with 1- and 4- $\mu \mathrm{m}$-thick PS layers (dots and solid circles, respectively).

neous Si nanostructure layer at a constant rate $(R)$ of approximately $1 \mu \mathrm{m} / \mathrm{min}$, which we confirmed by scanning electron microscope observations. By controlling the duration of the anodization, we prepared PS layers that were 1,2, 4, and 8 $\mu \mathrm{m}$ thick. Following the anodization, gold film was deposited as an electrode with the active device area being about 4 $\mathrm{mm}$ in diameter.

Figure 1 shows typical logarithmic $J-V$ characteristics for Au/PS junctions with 1- and 4- $\mu$ m-thick PS layers (dots and solid circles, respectively). From the lower current density region around $10^{-8} \mathrm{~A} / \mathrm{cm}^{2}$, we estimated the resistivity of the layer to be on the order of $10^{12} \Omega \mathrm{cm}$. This value shows that the porous layer can be regarded as an intrinsic semiconductor. For both samples $(1$ and $4 \mu \mathrm{m})$, the $J-V$ characteristics exhibit a power-law relationship, $J=\kappa V^{\nu}(\kappa$ is a proportionality factor), where $\nu$ increases from 1 to 4 and then decreases from 4 to almost 2 with increasing applied bias voltage. On the other hand, the current-thickness $(J-L)$ characteristics exhibit another power-law relationship, $J$ $=\eta L^{-\xi}$ ( $\eta$ is a proportionality factor), where $\xi$ increases from 1 to between 2 and 3 with increasing applied voltage. This behavior for both characteristics is similar to the SCLC with deep traps observed for single carrier injection into an intrinsic amorphous semiconductor. ${ }^{12,14}$ In the continuous state distribution of the $a$ semiconductor, the dominant level controlling the SCLC is situated close to the quasi-Fermi level and thus enables the determination of the density and distribution of localized states $[N(E)] .{ }^{8}$ To determine $N(E)$ from the logarithmic $J-V$ characteristics in the SCL region, we adopted the step-by-step method developed by den Böer. ${ }^{13}$ The density of states near the Fermi level can be expressed as

$$
N(E)=\frac{\epsilon\left(V_{2}-V_{1}\right)}{q L^{2} k T \ln \left(J_{2} / J_{1} V_{1} / V_{2}\right)},
$$

where $\left(J_{1}, V_{1}\right)$ and $\left(J_{2}, V_{2}\right)$ are points on the $J-V$ curve in Fig. 1, $\epsilon$ is a dielectric constant of the porous layer, $q$ is the electron charge, $L$ is the thickness of the porous layer, $k$ is

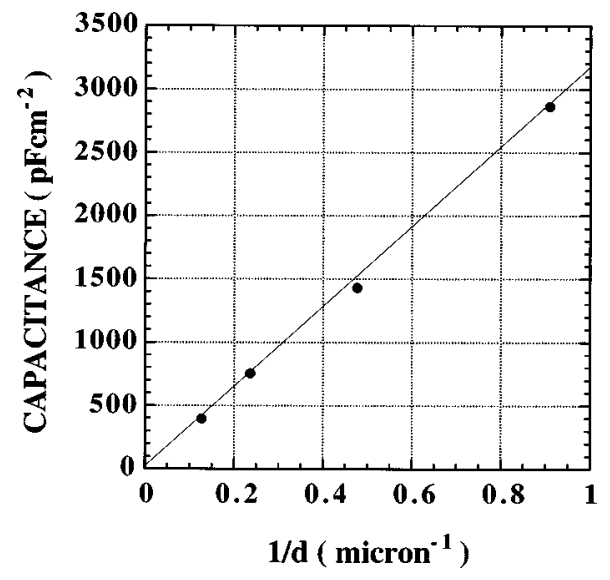

FIG. 2. Zero-bias capacitance for Au/PS junctions with different PS thicknesses. The fitted line can be obtained with the dielectric constant $\epsilon=3.6$.

the Boltzmann constant, and $T$ is temperature in degrees $\mathrm{K}$. Before we can determine $N(E)$, we need to determine the dielectric constant of the porous layer.

Figure 2 shows the thickness dependence of the zerobias capacitance of Au/PS devices. We can neglect the capacitance of the interface based on the Peng et al. results. ${ }^{15}$ In this case, the total capacitance is equal to the PS layer capacitance $\left(C_{\mathrm{PS}}\right)$, which is given by $C_{\mathrm{PS}}=\epsilon S / L$, where $S$ is the area of the diode. Measurements taken on devices with various PS layer thicknesses verify that the $C_{\mathrm{PS}}$ is proportional to $1 / L$ as shown in Fig. 2. We can, therefore, determine the dielectric constant $\epsilon$ to be $3.6 \epsilon_{0}$ ( $\epsilon_{0}$ is the dielectric constant of a vacuum), which agrees well with previously reported results for $80 \%$-porosity samples. ${ }^{4,16}$

We determined $N(E)$ for the 4- $\mu$ m-thick PS sample by applying Eq. (1) to the measured $J-V$ characteristics. Figure 3 shows $N(E)$ near the Fermi level determined by this stepby-step method. The points were obtained by applying Eq. (1) with $L=4 \mu \mathrm{m}, k T=26 \mathrm{meV}$, and steps of about $50 \mathrm{mV}$. The density of states near the Fermi level deduced here did

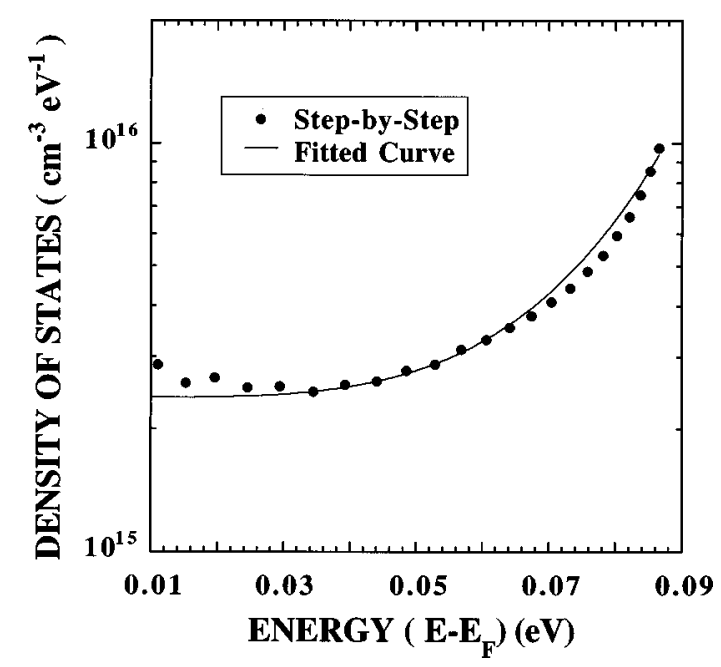

FIG. 3. The density of state distribution near the Fermi level determined by a step-by-step method (solid circles) and the theoretical curve (solid line) using a stretched exponential function. 


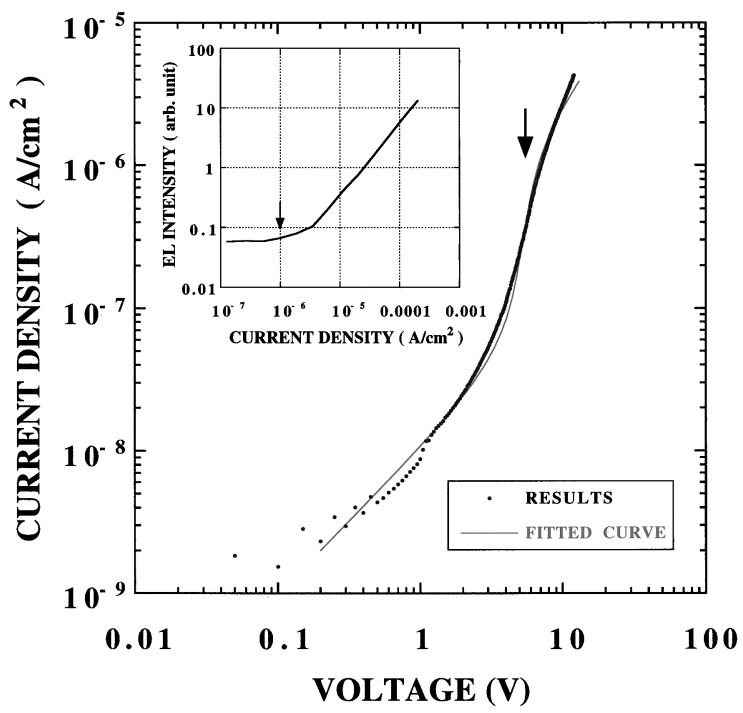

FIG. 4. Current-voltage $(J-V)$ characteristics of a Au/PS diode with a $1-\mu \mathrm{m}$-thick PS layer. The dotted line shows the theoretical curve of the $J-V$ characteristics. The inset shows the electroluminescence-current (EL-J) characteristics.

not form a simple exponential tail, but can be well described by a stretched exponential function:

$$
N(E)=\alpha \exp \left\{\left[\beta\left(E-E_{F}\right)\right]^{\gamma}\right\},
$$

where the data shown in Fig. 3 can be well fitted by $\alpha$ $=2.4 \times 10^{15}\left(\mathrm{~cm}^{-3} \mathrm{eV}^{-1}\right), \quad \beta=\left(k T_{t}\right)^{-1}=12.5\left(\mathrm{eV}^{-1}\right)$ with $T_{t}$ being a temperature parameter that characterizes the trap distribution, and $\gamma=4$. The steep rise in $N(E)$ toward the Fermi level might not represent the real density of states but appears to arise from an inaccuracy of this method, because this inaccuracy generally occurs in the evaluation of $N(E)$ using step-by-step or differential methods. ${ }^{17}$ The deduced $N(E)$, which is minimum at the center of the energy gap (Fermi level), is similar to the commonly observed U-shape trap distribution observed at the crystalline $\mathrm{Si}-\mathrm{SiO}_{2}$ interface ${ }^{18-21}$ or to the density of states observed in amorphous $\mathrm{Si}^{10}{ }^{10}$ The origin of $\gamma=4$ in the stretched exponential is not clearly understood; however, we consider that both the various energy levels originating from the size distribution of nanocrystallites and the coexistence of the tissue (amorphous) phase with nanocrystals generate the randomness expressed by a stretched exponential distribution.

Electroluminescence is a general concern when using this material; therefore, understanding the relation between EL intensity and a current density is critical. The inset of Fig. 4 shows the EL- $J$ characteristics of a Au/PS diode with a $1-\mu \mathrm{m}$-thick PS layer. Comparison of these EL- $J$ characteristics and $J-V$ characteristics (Fig. 4) clearly shows that EL occurred at the higher injection regime, above $10^{-6} \mathrm{~A} / \mathrm{cm}^{2}$, as indicated by arrows. This result is explained as follows: when the injected carrier concentration to the thermally generated carriers significantly increases, the $J-V$ characteristics of the device deviates from Ohmic behavior and holes begin to be injected into the porous layer, thereby significantly increasing the probability of electron-hole recombination (double-injection regime). Therefore, below the cur- rent density of $10^{-6} \mathrm{~A} / \mathrm{cm}^{2}$, where emission does not occur, we can consider electrons to be the dominant current carriers in this region (single-injection regime). Although the polarity of the dominant carrier injected into the porous layer is not yet known, $x$-ray absorption spectroscopy shows that the energy offset of the valence band is twice that of the conduction band in PS/Si junctions ${ }^{22}$ and thermopower measurements show the majority carriers are electrons. ${ }^{23}$

In the single-carrier injection region, the $J-V$ curve in the SCLC region, with the stretched exponential trap distribution as described in Eq. (2), can be calculated parametrically as

$$
\begin{aligned}
& V(\theta)=\left(\frac{\alpha e L_{0}^{2}}{\epsilon}\right) \int_{0}^{\theta} d x \frac{\exp \left[(\beta x)^{\gamma}\right]}{1+\exp (x / k T)} \\
& J(\theta)=\left(\frac{\alpha e^{2} n_{0} \mu L_{0}}{\epsilon}\right) \exp (\theta / k T) \int_{0}^{\theta} d x \frac{\exp \left[(\beta x)^{\gamma}\right]}{1+\exp (x / k T)},
\end{aligned}
$$

and the current-thickness $(J-L)$ curve as

$$
\begin{aligned}
L(\theta)= & \left(\frac{\epsilon V_{0}}{\alpha e}\right)^{1 / 2}\left[\int_{0}^{\theta} d x \frac{\exp \left[(\beta x)^{\gamma}\right]}{1+\exp (x / k T)}\right]^{-1 / 2}, \\
J(\theta)= & \left(\frac{\alpha e^{3} n_{0}^{2} \mu^{2} V_{0}}{\epsilon}\right)^{1 / 2} \exp (\theta / k T) \\
& \times\left[\int_{0}^{\theta} d x \frac{\exp \left[(\beta x)^{\gamma}\right]}{1+\exp (x / k T)}\right]^{1 / 2},
\end{aligned}
$$

where the parameter $\theta$ is equal to $F-E_{f}, F$ is the quasiFermi energy, $E_{f}$ is the Fermi energy, $n_{0}$ is a thermal equilibrium free-electron concentration, $\mu$ is the effective mobility, $L_{0}$ is the constant thickness, and $V_{0}$ is the constant applied voltage. As a check, in the case of $\gamma=1$, which corresponds to the exponential trap distribution, ${ }^{8}$ we can derive the correct dependence of SCLC as $J \propto V^{m+1} / L^{2 m+1}$ (with $\left.m=T_{t} / T\right)$ by the elimination of the parameter $\theta$ after the integration.

By using parametric plots through the combinations with Eqs. (3) and (4), or (5) and (6), we obtain the theoretical $J-V$ curves or $J-L$ curves for the density of states given by Eq. (2). The dotted line in Fig. 4 shows the theoretical curve of the $J-V$ characteristic for the sample with a $1-\mu \mathrm{m}$-thick PS layer. The best fit of this curve was obtained using the parameters $\alpha=6 \times 10^{16}\left(\mathrm{~cm}^{-3} \mathrm{eV}^{-1}\right)$ and $n_{0} \mu=1.15$ $\times 10^{6}\left(\mathrm{~cm}^{-1} \mathrm{~V}^{-1} \mathrm{~s}^{-1}\right)$. The reason why the magnitude of $N(E)$ of a thin layer is higher than that of a thick layer can be understood in terms of a higher density of states in the surface region - the SCLC probes the entire volume distribution. The agreement between the experimental result and the theoretical curve strongly implies that the current flow is entirely controlled by localized states situated at the quasiFermi level. The deviation of the theoretical curves and the experimental results at the region higher than $10^{-6} \mathrm{~A} / \mathrm{cm}^{2}$ may originate from the inaccuracy of the fitting function and/or the contribution of hole current (i.e., the deviation from the single-injection analysis).

The $J-L$ relation given by Eqs. (5) and (6) was also confirmed for various thicknesses $(1-8 \mu \mathrm{m})$ of the porous 


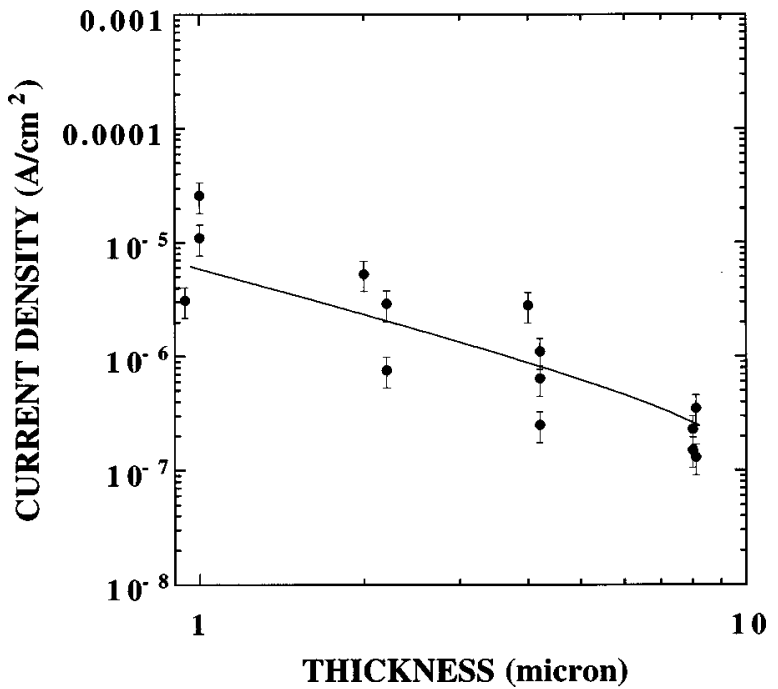

FIG. 5. Experimental current density (solid circles) versus thickness $(J-T)$ characteristics at a constant applied potential of $30 \mathrm{~V}$. The solid line shows the theoretical curve of the $J-T$ characteristics.

layer. Figure 5 shows the experimental plots (solid circles) for the $J-L$ characteristics at a constant applied potential of $30 \mathrm{~V}$. The solid lines show the theoretical curves obtained from Eqs. (2), (5), and (6) using the same parameters described above $\left[\alpha=2.4 \times 10^{15}\left(\mathrm{~cm}^{-3} \mathrm{eV}^{-1}\right), \quad \beta=12.5\right.$ $\left(\mathrm{eV}^{-1}\right)$, and $\left.\gamma=4\right]$. The theoretical curves well explain the experimental plots of the $J-L$ relation. Such agreement suggests that the SCLC dominates the carrier conduction behavior.

\section{DISCUSSION}

Generally, for trap free or shallow traps with a singleenergy level, the behavior of the SCLC obeys $J \propto V^{2} / L^{3}$, which was generally applied for PS layers. ${ }^{15}$ However, the logarithmic $J-V$ characteristics reported here and elsewhere ${ }^{3,15}$ generally show the power of $V$ increasing from 1 to 3 or 4 , depending on the applied potential, and therefore, cannot be fitted by the simple $V^{2} / L^{3}$ relationship. If we take a certain region from the $J-V$ data, we might find a region that obeys the $V^{2} / L^{3}$ relationship. However, the assumption of trap free is not realistic for nanostructural PS. Moreover, our experimental results indicate the existence of deep traps (Fig. 3). The agreement between the $J-V$ (and also $J-T$ ) data and the above analysis in the whole range of applied potential (and also thickness) confirms that PS contains localized states on the order of $10^{15}\left(\mathrm{~cm}^{-3} \mathrm{eV}^{-1}\right)$, which make the characteristic current flow as shown in Fig. 1.

We used the parameters $n_{0} \mu \approx 10^{6}$ to fit the experimental results. Using a previously reported value of $\mu$ $\left[\approx 10^{-3}\left(\mathrm{~cm}^{2} \mathrm{~V}^{-1} \mathrm{~s}^{-1}\right)\right]$ for nanostructural PS, ${ }^{23,24}$ we determined the intrinsic carrier concentration $\left(n_{0}\right)$ of PS to be on the order of $10^{9} \mathrm{~cm}^{-3}$. If we also take porosity into account, the correct intrinsic carrier concentration becomes on the order of $10^{10} \mathrm{~cm}^{-3}$, which is similar to that of bulk Si crystal. ${ }^{25}$ The intrinsic carrier density depends on the band gap; because PS is considered a wide-band-gap material, its intrinsic carrier density is expected to be smaller than that of bulk $\mathrm{Si}$ crystal. The effect of the quantum confinement on the intrinsic carrier density and on the transport is not yet clear; however, the energy gap determined by carrier transport may not be as wide as the optical band gap. ${ }^{26}$

Porous Si with $80 \%$ porosity has a large surface area of the order of $200\left(\mathrm{~m}^{2} / \mathrm{cm}^{3}\right) .{ }^{27}$ By converting the bulk density of states into the surface states using this value, we obtain the interface state density distribution to be about $10^{9}\left(\mathrm{~cm}^{-2} \mathrm{eV}^{-1}\right)$. The magnitude of this state is similar to the number of $P_{b}$ centers obtained by our electron spin resonance measurements ${ }^{28}$ and to the trap density [about $\left.10^{9}\left(\mathrm{~cm}^{-2} \mathrm{eV}^{-1}\right)\right]$ estimated by Cadet et al. using transient current measurements. ${ }^{29}$ It is not clear if $N(E)$ obtained here originates from $P_{b}$ centers; however, not only our results but also other reports ${ }^{29,30}$ show th correlation between the trap concentrations and $P_{b}$ centers.

\section{CONCLUSION}

In conclusion, we determined the density of state distribution $[N(E)]$ of Au/PS junctions using a step-by-step method. The deduced $N(E)$ can be fitted well by a stretched exponential function. Similarly, both the current-voltage and the current-thickness characteristics of PS can also be fitted well with the theoretical curves obtained by space-chargelimited-current analysis. The agreement between the experimental results and the theoretical curves strongly suggests that PS contains localized states on the order of $10^{15}\left(\mathrm{~cm}^{-3} \mathrm{eV}^{-1}\right)$ situated at the quasi-Fermi level, which dominates the electric properties of PS. Important problems related to the origin of the localized states situated near the Fermi level remain unresolved.

\section{ACKNOWLEDGMENTS}

The authors are grateful to P. M. Fauchet, L. T. Canham, M. Kondo, and S. V. Nair for valuable discussions and assistance with this work.

${ }^{1}$ L. T. Canham, Appl. Phys. Lett. 57, 1046 (1990).

${ }^{2}$ N. Koshida and H. Koyama, Appl. Phys. Lett. 60, 347 (1992).

${ }^{3}$ T. Futagi, T. Matsumoto, M. Katsuno, Y. Ohta, H. Mimura, and K. Kitamura, Appl. Phys. Lett. 63, 1209 (1993).

${ }^{4}$ P. Steiner, F. Kozlowsky, and W. Lang, Appl. Phys. Lett. 62, 2700 (1993).

${ }^{5}$ A. Loni, A. J. Simons, T. I. Cox, P. D. J. Calcott, and L. T. Canham, Electron. Lett. 31, 1288 (1995).

${ }^{6}$ K. D. Hirschman, L. Tsybeskov, S. P. Duttagupta, and P. M. Fauchet, Nature (London) 384, 338 (1996).

${ }^{7}$ R. T. Collins, P. M. Fauchet, and M. A. Tischler, Phys. Today 24, (1997).

${ }^{8}$ M. A. Lampert and P. Mark, Current Injection in Solids (Academic, New York, 1970).

${ }^{9}$ A. Maden, P. G. Le Comber, and W. E. Spear, J. Non-Cryst. Solids 20, 239 (1976).

${ }^{10}$ M. Hirose, T. Suzuki, and G. H. Dohler, Appl. Phys. Lett. 34, 234 (1979).

${ }^{11}$ J. D. Cohen, D. V. Lang, and J. P. Harbison, Phys. Rev. Lett. 45, 197 (1980).

${ }^{12}$ K. D. Mackenzie, P. G. Le Comber, and W. E. Spear, Philos. Mag. B 46, 377 (1982).

${ }^{13}$ W. Den Böer, J. Phys. (Paris), Colloq. 42, 451 (1981).

${ }^{14}$ S. Nespurek and J. Sworakowski, J. Appl. Phys. 51, 2098 (1980).

${ }^{15}$ C. Peng, D. Hirschman, and P. M. Fauchet, J. Appl. Phys. 80, 295 (1996).

${ }^{16}$ M. Adam, Z. J. Horvath, I. Barsony, L. Szolgyemy, E. Vazsonyi, and V. V. Tuyen, Thin Solid Films 225, 266 (1995).

${ }^{17}$ K. Shimakawa and Y. Katsuma, J. Appl. Phys. 60, 1417 (1986). 
${ }^{18}$ H. Sakaki, K. Hoh, and T. Sugano, IEEE Trans. Electron Devices ED-17, 892 (1970).

${ }^{19}$ T. Sakurai and T. Sugano, J. Appl. Phys. 52, 2889 (1981).

${ }^{20}$ E. H. Poindexter, G. J. Gerardi, M.-E. Rueckel, P. J. Caplan, N. M. Johnson, and D. K. Biegelsen, J. Appl. Phys. 56, 2844 (1984).

${ }^{21}$ R. R. Razouk and B. E. Deal, J. Electrochem. Soc. 126, 1573 (1979).

${ }^{22}$ T. van Buuren, T. Tiedje, J. R. Dahn, and B. M. Way, Appl. Phys. Lett. 63, 2911 (1993).

${ }^{23}$ M. Ben-Chorin, S. Grebner, F. Wang, R. Schwarz, A. Nikolov, and F. Koch, Mater. Res. Soc. Symp. Proc. 358, 575 (1994).

${ }^{24}$ É. A. Lebedev, G. Polisskii, and V. Perova-Koch, Semiconductors 30, 772 (1996).
${ }^{25}$ S. M. Sze, Physics of Semiconductor Devices, 2nd Ed. (Wiley, New York, 1981).

${ }^{26}$ J. Kocka, A. Fejfar, and I. Pelant, Phys. Status Solidi B 190, 27 (1995).

${ }^{27}$ R. Herino, G. Bomchil, K. Barla, C. Bertrand, and J. L. Ginoux, J. Electrochem. Soc. 134, 1994 (1987).

${ }^{28}$ T. Matsumoto, M. Kondo, S. V. Nair, and Y. Masumoto, J. Non-Cryst. Solids 227-230, 320 (1998).

${ }^{29}$ C. Cadet, D. Deresmes, D. Vuillaume, and D. Stievenard, Appl. Phys. Lett. 64, 2827 (1994)

${ }^{30}$ A. Grosman and C. Ortega, in Properties of Porous Silicon, edited by L. T. Canham (INSPEC, London), pp. 328-335. 